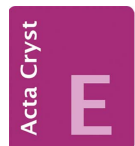

CRYSTALLOGRAPHIC COMMUNICATIONS

ISSN 2056-9890

Received 18 April 2017

Accepted 3 May 2017

Edited by M. Weil, Vienna University of

Technology, Austria

Keywords: apremilast; PDE4; psoriatic arthritis; crystal structure; hydrogen bonding; solvatomorph.

CCDC reference: 1504229

Supporting information: this article has supporting information at journals.iucr.org/e

\section{Crystal structure of an apremilast ethanol hemisolvate hemihydrate solvatomorph}

\author{
Yun-Deng $\mathrm{Wu}^{\mathrm{a} *}{ }^{\mathrm{a}}$ Xiao-Hong Liu, ${ }^{\mathrm{a}}$ Jian $\mathrm{Xu},{ }^{\mathrm{a}}$ Si-Han Zhang, ${ }^{\mathrm{a}}$ Kun Shen, ${ }^{\mathrm{a}}$ Ling Sun, \\ Yong-Mei He, ${ }^{a}$ Yan $\mathrm{Ma}^{\mathrm{a}}$ and $\mathrm{Ai}-\mathrm{Hua}$ Zhang ${ }^{\mathrm{a}}$
}

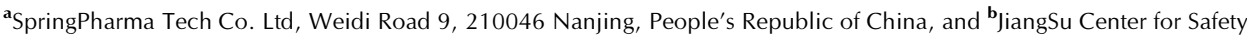 \\ Evaluation of Drugs, Majia Street 26, 210009 Nanjing, People's Republic of China. *Correspondence e-mail: \\ wuyundeng@hotmail.com
}

The title compound, $\mathrm{C}_{22} \mathrm{H}_{24} \mathrm{~N}_{2} \mathrm{O}_{7} \mathrm{~S} \cdot 0.5 \mathrm{C}_{2} \mathrm{H}_{5} \mathrm{OH} \cdot 0.5 \mathrm{H}_{2} \mathrm{O}$ \{systematic name: (S)-4-acetamido-2-[1-(3-ethoxy-4-methoxyphenyl)-2-(methylsulfonyl)ethyl]isoindoline-1,3-dione ethanol hemisolvate hemihydrate\}, is a novel solvatomorph of apremilast (AP), which is an inhibitor of phosphodiesterase 4 (PDE4) and is indicated for the treatment of adult patients with active psoriatic arthritis. The asymmetric unit contains one molecule of AP and disordered molecules of ethanol and water, both with half occupancy. The dihedral angle between the planes of the phenyl ring and the isoindole ring is $67.9(2)^{\circ}$. Extensive intra- and intermolecular hydrogen bonds help to stabilize the molecular conformation and sustain the crystal packing.

\section{Chemical context}

Analogues of thalidomide have been reported to possibly enhance tumor necrosis factor alpha (TNF $\alpha)$ inhibitory activity (Corral et al., 1996; Muller et al., 1996) and phosphodiesterase type 4 (PD4) inhibition (Muller et al., 1998), hence showing potential for the treatment of inflammatory diseases (de Brito et al., 1997). Among these substances are phenethylsulfones substituted in the $\alpha$ position to the phenyl group with a 1-oxoisoindoline or 1,3-dioxoisoindoline group that can reduce the levels of TNF $\alpha$ in a mammal. Typical embodiments are $(S)$-2-[1-(3-ethoxy-4-methoxyphenyl)-2-(methylsulfonyl)ethyl]-4-acetamidoisoindoline-1,3-dione] with the generic name apremilast (AP), which is an inhibitor of phosphodiesterase 4 (PDE4) and is indicated for the treatment of adult patients with active psoriatic arthritis (Gottlieb et al., 2008; Man et al., 2009; Duplantier et al., 1996). In our previous studies, we reported three solvatomorphs of AP with ethyl acetate, toluene and dichloromethane, respectively (Wu et al., 2017). However, these three solvates exhibit toxicity, in particular the solvates of toluene and dichloromethane, which clearly limits the possibility of these compounds being developed into drugs. In a continuation of our work, a novel solvatomorph of AP with ethanol and water solvents in the molar ratio 1:0.5:0.5 was prepared and its crystal structure determined. This solvatomorph of AP appears to be suitable for development into a powerful drug, showing much lower toxicity than the solvatomorphs of ethyl acetate, toluene and dichloromethane. 
<smiles>CCCCCCCCCCO</smiles>

\section{Structural commentary}

The title solvatomorph (I) crystallizes in the same space group $\left(P 4_{1} 2_{1} 2\right)$ as the other three structurally characterized solvatomorphs of ethyl acetate, toluene and dichloromethane (Wu et al., 2017). The structures of the molecular components of (I) are shown in Fig. 1. The asymmetric unit comprises one molecule of AP and one solvent molecule each of ethanol and water, both being disordered about a twofold rotation axis (occupancy for both solvent molecules $=0.5$ ). A space-filling drawing of the structure is given in Fig. 2, emphasizing the

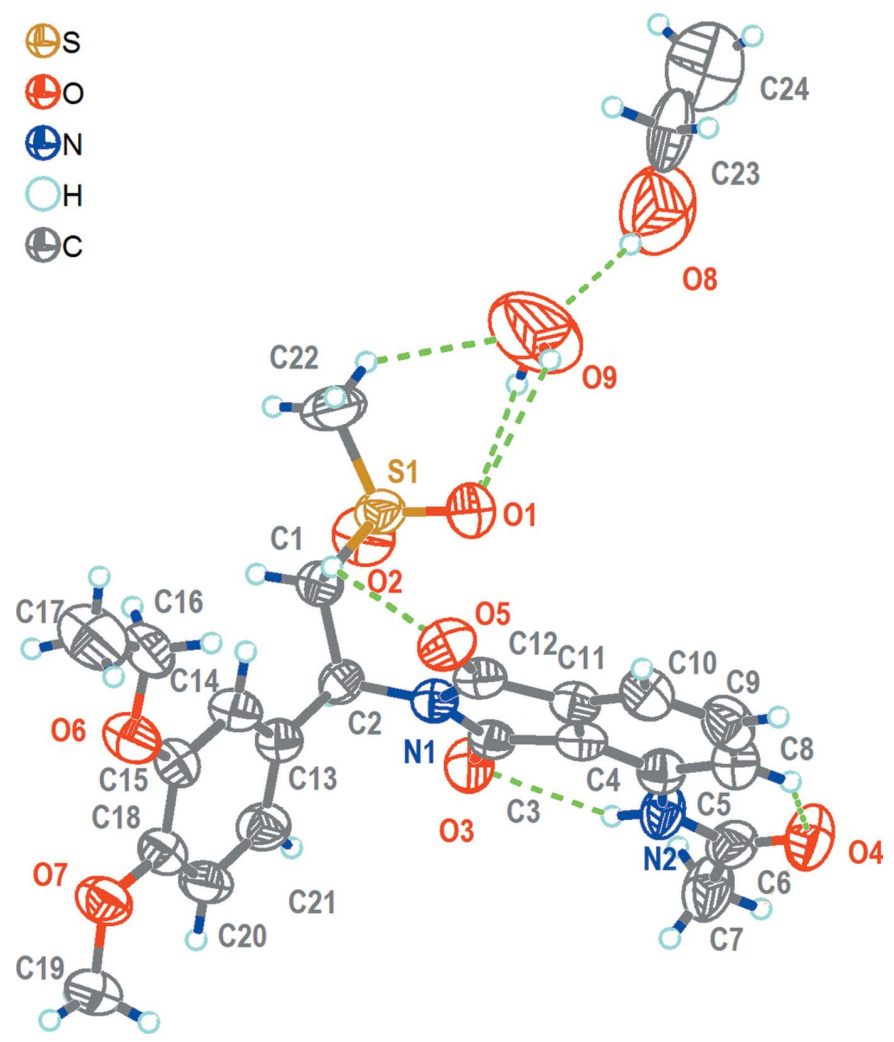

Figure 1

The structures of the molecular components in (I). Displacement ellipsoids are drawn at the $50 \%$ probability level. Hydrogen bonds are shown as dashed lines.
Table 1

Hydrogen-bond geometry $\left(\AA{ }^{\circ}\right)$.

\begin{tabular}{lllll}
\hline$D-\mathrm{H} \cdots A$ & $D-\mathrm{H}$ & $\mathrm{H} \cdots A$ & $D \cdots A$ & $D-\mathrm{H} \cdots A$ \\
\hline $\mathrm{N} 2-\mathrm{H} 2 \cdots \mathrm{O} 3$ & 0.86 & 2.31 & $2.986(7)$ & 136 \\
$\mathrm{O} 8-\mathrm{H} 8 A \cdots \mathrm{O} 9$ & 0.92 & 2.30 & $3.20(3)$ & 166 \\
$\mathrm{O} 9-\mathrm{H} 9 B \cdots \mathrm{O} 1$ & 0.89 & 2.54 & $2.994(17)$ & 112 \\
$\mathrm{C} 8-\mathrm{H} 8 \cdots \mathrm{O} 4$ & 0.93 & 2.30 & $2.894(9)$ & 121 \\
$\mathrm{C} 1-\mathrm{H} 1 A \cdots \mathrm{O} 5$ & 0.97 & 2.45 & $3.068(8)$ & 121 \\
$\mathrm{C} 1-\mathrm{H} 1 A \cdots 5^{\mathrm{i}}$ & 0.97 & 2.32 & $3.172(8)$ & 147 \\
$\mathrm{C} 1-\mathrm{H} 1 B \cdots \mathrm{O}^{\text {ii }}$ & 0.97 & 2.56 & $3.524(9)$ & 172 \\
$\mathrm{C} 14-\mathrm{H} 14 \cdots 5^{\mathrm{i}}$ & 0.93 & 2.49 & $3.415(8)$ & 178 \\
$\mathrm{C} 19-\mathrm{H} 19 C \cdots \mathrm{O} 4^{\text {iii }}$ & 0.96 & 2.61 & $3.567(10)$ & 173 \\
$\mathrm{C} 20-\mathrm{H} 20 \cdots \mathrm{O} 2^{\mathrm{ii}}$ & 0.93 & 2.46 & $3.370(9)$ & 166 \\
$\mathrm{C} 22-\mathrm{H} 22 C \cdots \mathrm{O} 3^{\text {iv }}$ & 0.96 & 2.44 & $3.088(9)$ & 124 \\
$\mathrm{C} 22-\mathrm{H} 22 C \cdots \mathrm{O} 9$ & 0.96 & 2.61 & $3.36(2)$ & 136 \\
\hline
\end{tabular}

Symmetry codes: (i) $y, x,-z+1$; (ii) $x+\frac{1}{2},-y+\frac{3}{2},-z+\frac{3}{4}$; (iii) $-y+\frac{5}{2}, x+\frac{1}{2}, z+\frac{1}{4}$; (iv) $-y+\frac{3}{2}, x-\frac{1}{2}, z+\frac{1}{4}$.

positions of the solvent molecules in the crystal structure. The bond lengths and angles in the AP molecule are in normal ranges and very similar to those in the previous three solvatomorphs (Wu et al., 2017). The same applies to the dihedral

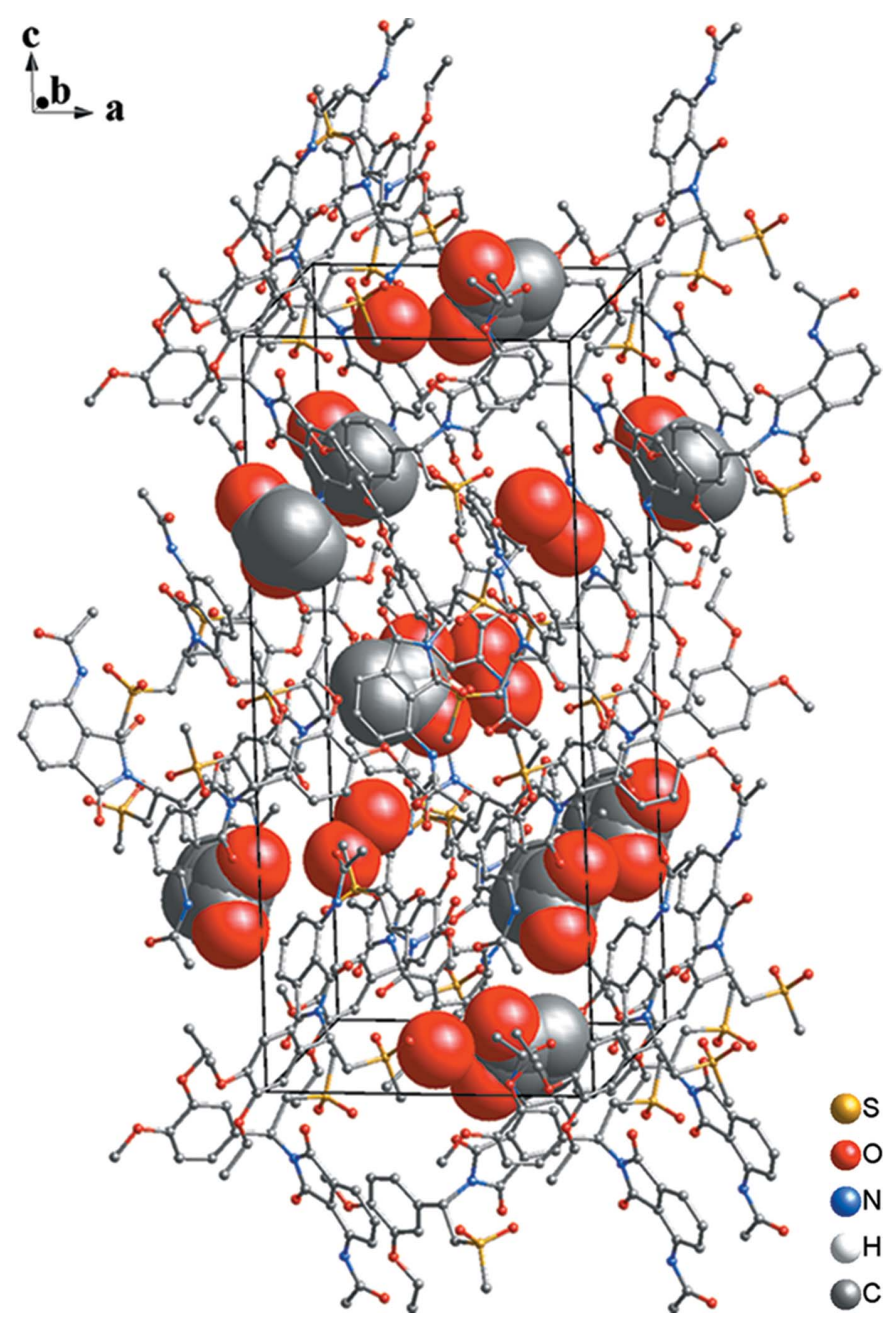

Figure 2

The unit cell of (I), with the solvent molecules shown in space-filling mode. [See Table 1 for symmetry codes.] 


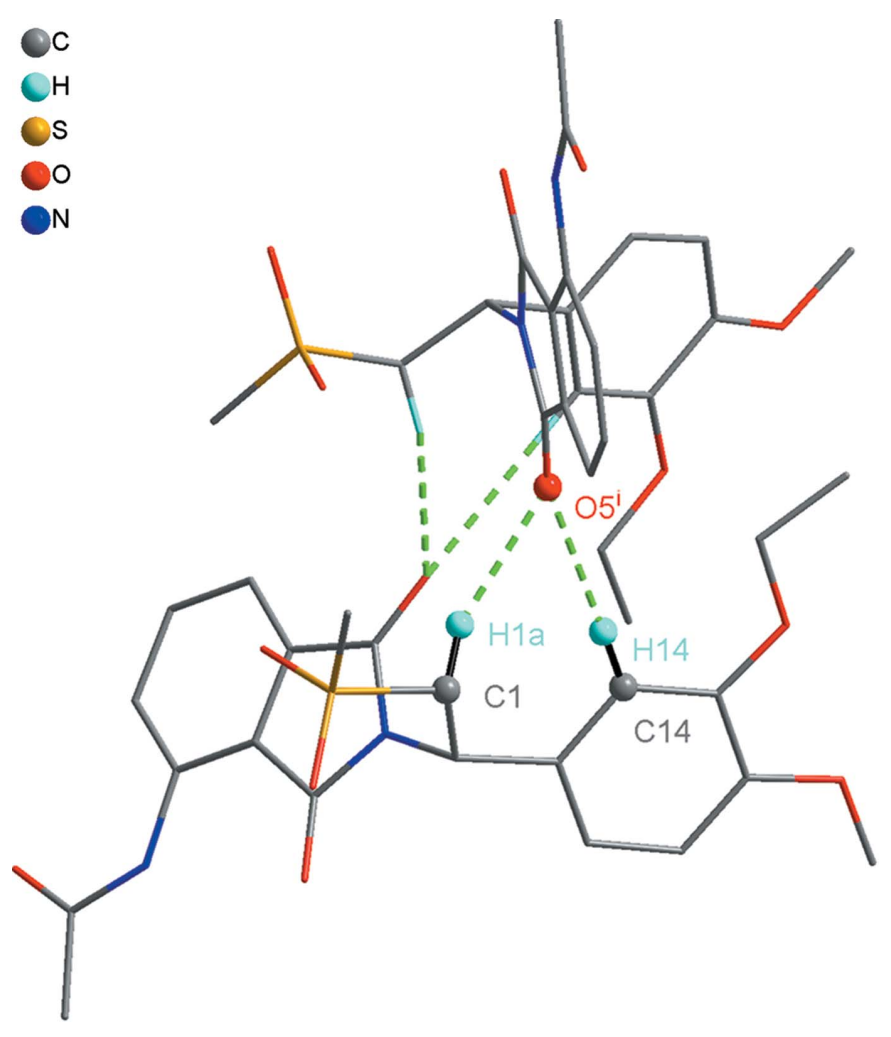

Figure 3

$(\mathrm{AP})_{2}$ dimers with an $R_{2}^{1}(7)$ motif formed by $\mathrm{C}-\mathrm{H} \cdots \mathrm{O}$ hydrogen bonds. [See Table 1 for symmetry code.]

angle between the phenyl (C13-C20) and isoindole (C3-C5/ $\mathrm{C} 8-\mathrm{C} 12 / \mathrm{N} 1)$ rings, which is $67.9(2)^{\circ}$ in the title structure. The conformation of the AP molecule is stabilized by several intramolecular hydrogen bonds of types $\mathrm{N}-\mathrm{H} \cdots \mathrm{O}$ and $\mathrm{C}-$ H... (Table 1).

\section{Supramolecular features}

An extensive network of intermolecular hydrogen-bonding interactions exists in the crystal structure (Figs. 1, 2-4; Table 1).
The water molecule $(\mathrm{O} 9)$ is hydrogen-bonded to the AP molecule by $\mathrm{C} 22-\mathrm{H} 22 C \cdots \mathrm{O} 9$ and $\mathrm{O} 9-\mathrm{H} 9 B \cdots \mathrm{O} 1$ interactions and likewise is bonded by an $\mathrm{O} 8-\mathrm{H} 8 A \cdots \mathrm{O} 9$ interaction to the ethanol solvent molecule. As well as these hydrogen bonds involving the solvent molecules, there are interactions between AP molecules. Two AP molecules are arranged into a dimer with an $R_{2}^{1}(7)$ motif (Fig. 3) by C$\mathrm{H}$... O hydrogen bonds, and a zipper-like chain including $R_{2}^{2}(18)$ motifs (Fig. 4) is formed parallel to the $a$ axis by additional $\mathrm{C}-\mathrm{H} \cdot$. O hydrogen bonds.

\section{Synthesis and crystallization}

AP was prepared according to a literature protocol (Muller et $a l ., 2006,2008 a, b)$. A $100 \mathrm{ml}$ round-bottomed flask equipped with a magnetic stirring bar was charged with a solution of (S)-1-(3-ethoxy-4-methoxyphenyl)-2-methylsulfonylethanamine $N$-acetyl leucine salt $(5.0 \mathrm{~g}, 11.2 \mathrm{mmol}, 1.0 \mathrm{eq})$ and 3-acetamidophthalic anhydride $(2.42 \mathrm{~g}, 11.8 \mathrm{mmol}, 1.05 \mathrm{eq})$ to which glacial acetic acid $(50 \mathrm{ml})$ was added. The mixture was refluxed for $16 \mathrm{~h}$ and then cooled to room temperature. The solvent was removed in vacuo, and the residue was dissolved in ethyl acetate. The resulting solution was washed with water $(2 \times 50 \mathrm{ml})$, saturated aqueous sodium bicarbonate $(2 \times 50 \mathrm{ml})$, brine $(2 \times 50 \mathrm{ml})$, and dried over anhydrous sodium sulfate. The solvents were evaporated in vacuo, and the obtained AP recrystallized from an ethanol/acetone mixture $(2: 1, v / v)$. Single crystals of (I) were obtained by slow evaporation of an AP-saturated solution from an $N, N$-dimethylformamide/ethanol/water mixture $(1: 10: 2, v / v / v)$, at room temperature over 90 days.

\section{Refinement}

Crystal data, data collection and structure refinement details are summarized in Table 2. Hydrogen atoms bound to nitrogen or carbon atoms were placed in calculated positions $(\mathrm{N}-$ $\mathrm{H}=0.87, \mathrm{C}-\mathrm{H}=0.93-0.98 \AA$ ) and constrained to ride on their carrier atoms $\left[U_{\text {iso }}(\mathrm{H})=1.2 U_{\text {eq }}(\mathrm{C}, \mathrm{N})\right.$ or $\left.1.5 U_{\text {eq }}\left(\mathrm{C}_{\text {methyl }}\right)\right]$.

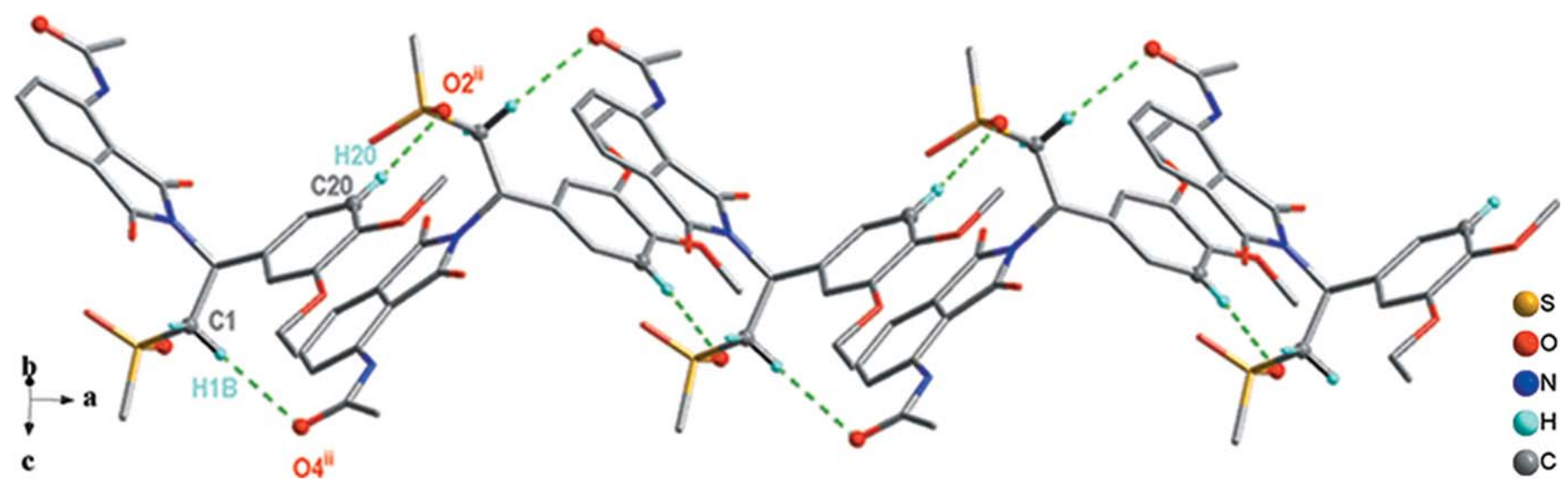

Figure 4

$\mathrm{C} 1-\mathrm{H} 1 B \cdots \mathrm{O} 4^{\mathrm{ii}}$ and $\mathrm{C} 20-\mathrm{H} 20 \cdots \mathrm{O} 2^{\mathrm{ii}}$ hydrogen bonds incorporating $R_{2}^{2}(18)$ motifs expand the structure parallel to the $a$-axis direction. [See Table 1 for symmetry codes.] 
Table 2

Experimental details.

\begin{tabular}{|c|c|}
\hline \multicolumn{2}{|l|}{ Crystal data } \\
\hline Chemical formula & $\mathrm{C}_{22} \mathrm{H}_{24} \mathrm{~N}_{2} \mathrm{O}_{7} \mathrm{~S} \cdot 0.5 \mathrm{C}_{2} \mathrm{H}_{6} \mathrm{O} \cdot 0.5 \mathrm{H}_{2} \mathrm{O}$ \\
\hline$M_{\mathrm{r}}$ & 492.53 \\
\hline Crystal system, space group & Tetragonal, $P 4_{1} 2{ }_{1} 2$ \\
\hline Temperature (K) & 298 \\
\hline$a, c(\AA)$ & 12.9905 (18), $29.942(6)$ \\
\hline$V\left(\AA^{3}\right)$ & $5052.8(17)$ \\
\hline$Z$ & 8 \\
\hline Radiation type & Мо $K \alpha$ \\
\hline$\mu\left(\mathrm{mm}^{-1}\right)$ & 0.18 \\
\hline Crystal size $(\mathrm{mm})$ & $0.3 \times 0.3 \times 0.2$ \\
\hline \multicolumn{2}{|l|}{ Data collection } \\
\hline Diffractometer & Bruker P4 \\
\hline $\begin{array}{l}\text { No. of measured, independent and } \\
\text { observed }[I>2 \sigma(I)] \text { reflections }\end{array}$ & $9575,4390,2811$ \\
\hline$R_{\text {int }}$ & 0.079 \\
\hline$(\sin \theta / \lambda)_{\max }\left(\AA^{-1}\right)$ & 0.592 \\
\hline \multicolumn{2}{|l|}{ Refinement } \\
\hline$R\left[F^{2}>2 \sigma\left(F^{2}\right)\right], w R\left(F^{2}\right), S$ & $0.066,0.183,1.05$ \\
\hline No. of reflections & 4390 \\
\hline No. of parameters & 329 \\
\hline No. of restraints & 30 \\
\hline $\mathrm{H}$-atom treatment & $\mathrm{H}$-atom parameters constrained \\
\hline$\Delta \rho_{\max }, \Delta \rho_{\min }\left(\mathrm{e} \AA^{-3}\right)$ & $0.62,-0.30$ \\
\hline Absolute structure & $\begin{array}{l}\text { Flack } x \text { determined using } 833 \\
\quad \text { quotients }\left[\left(I^{+}\right)-\left(I^{-}\right)\right] /\left[\left(I^{+}\right)+\left(I^{-}\right)\right] \\
\quad \text { (Parsons } \text { et al., 2013) }\end{array}$ \\
\hline Absolute structure parameter & $0.15(8)$ \\
\hline
\end{tabular}

Computer programs: APEX2 and SAINT (Bruker, 2009), SHELXT (Sheldrick, 2015a), SHELXL2014 (Sheldrick, 2015b) and OLEX2 (Dolomanov et al., 2009).

Hydrogen atoms bound to oxygen atoms were deduced from difference-Fourier maps and their positions relative to donor and possible acceptor atoms. They were refined with $U_{\text {iso }}(\mathrm{H})=$ $1.5 U_{\text {eq }}(\mathrm{O})$. The solvent ethanol and water molecules are disordered about a twofold rotation axis and were refined with an occupancy of 0.5 . To get reasonable shape and displacement parameters for both molecules, they were treated with
DFIX, RIGU and ISOR restraints in SHELXL2014 (Sheldrick, 2015b).

\section{References}

Brito, F. de, Souness, J. E. \& Warne, P. J. (1997). Emerg. Drugs, 2, 249 268.

Bruker (2009). APEX2 and SAINT. Bruker AXS Inc., Madison, Wisconsin, USA.

Corral, L. G., Muller, G. W., Moreira, A. L., Chen, Y., Wu, M., Stirling, D. I. \& Kaplan, G. (1996). Mol. Med. 2, 506-515.

Dolomanov, O. V., Bourhis, L. J., Gildea, R. J., Howard, J. A. K. \& Puschmann, H. (2009). J. Appl. Cryst. 42, 339-341.

Duplantier, A. J., Biggers, M. S., Chambers, R. J., Cheng, J. B., Cooper, K., Damon, D. B., Eggler, J. F., Kraus, K. G., Marfat, A., Masamune, H., Pillar, J. S., Shirley, J. T., Umland, J. P. \& Watson, J. W. (1996). J. Med. Chem. 39, 120-125.

Gottlieb, A. B., Strober, B., Krueger, J. G., Rohane, P., Zeldis, J. B., Hu, C. C. \& Kipnis, C. (2008). Curr. Med. Res. Opin. 24, 1529-1538.

Man, H. W., Schafer, P., Wong, L. M., Patterson, R. T., Corral, L. G., Raymon, H., Blease, K., Leisten, J., Shirley, M. A., Tang, Y., Babusis, D. M., Chen, R., Stirling, D. \& Muller, G. W. (2009). J. Med. Chem. 52, 1522-1524.

Muller, G. W., Corral, L. G., Shire, M. G., Wang, H., Moreira, A. L., Kaplan, G. \& Stirling, D. I. (1996). J. Med. Chem. 39, 3238-3240.

Muller, G. W., Schafer, P. H., Man, H. W. \& Ge, C. (2008a). US Patent No. 7427638.

Muller, G. W., Schafer, P. H., Man, H.-W., Ge, C. \& Xu, J. (2008b). WO Patent No. 2009120167.

Muller, G. W., Schafer, P. \& Rohane, P. (2006). US Patent No. 2006183787.

Muller, G. W., Shire, M. G., Wong, L. M., Corral, L. G., Patterson, R. T., Chen, Y. \& Stirling, D. I. (1998). Bioorg. Med. Chem. Lett. 8, 2669-2674.

Parsons, S., Flack, H. D. \& Wagner, T. (2013). Acta Cryst. B69, 249 259.

Sheldrick, G. M. (2015a). Acta Cryst. A71, 3-8.

Sheldrick, G. M. (2015b). Acta Cryst. C71, 3-8.

Wu, Y.-D., Zhang, X.-L., Liu, X.-H., Xu, J., Zhang, M., Shen, K., Zhang, S.-H., He, Y.-M., Ma, Y. \& Zhang, A.-H. (2017). Acta Cryst. C73, 305-313. 


\section{supporting information}

Acta Cryst. (2017). E73, 821-824 [https://doi.org/10.1107/S2056989017006661]

\section{Crystal structure of an apremilast ethanol hemisolvate hemihydrate solvatomorph}

Yun-Deng Wu, Xiao-Hong Liu, Jian Xu, Si-Han Zhang, Kun Shen, Ling Sun, Yong-Mei He, Yan

Ma and Ai-Hua Zhang

Computing details

Data collection: APEX2 (Bruker, 2009); cell refinement: SAINT (Bruker, 2009); data reduction: SAINT (Bruker, 2009); program(s) used to solve structure: SHELXT (Sheldrick, 2015a); program(s) used to refine structure: SHELXL2014 (Sheldrick, 2015b); molecular graphics: OLEX2 (Dolomanov et al., 2009); software used to prepare material for publication: OLEX2 (Dolomanov et al., 2009).

(S)-4-Acetamido-2-[1-(3-ethoxy-4-methoxyphenyl)-2-(methylsulfonyl)ethyl]isoindoline-1,3-dione ethanol hemisolvate hemihydrate

\section{Crystal data}

$\mathrm{C}_{22} \mathrm{H}_{24} \mathrm{~N}_{2} \mathrm{O}_{7} \mathrm{~S} \cdot 0.5 \mathrm{C}_{2} \mathrm{H}_{6} \mathrm{O} \cdot 0.5 \mathrm{H}_{2} \mathrm{O}$

$M_{r}=492.53$

Tetragonal, $P 4_{1} 2_{1} 2$

$a=12.9905(18) \AA$

$c=29.942(6) \AA$

$V=5052.8(17) \AA^{3}$

$Z=8$

$F(000)=2080$

Data collection

Bruker P4

diffractometer

$\omega$ scans

9575 measured reflections

4390 independent reflections

2811 reflections with $I>2 \sigma(I)$

$R_{\text {int }}=0.079$

\section{Refinement}

Refinement on $F^{2}$

Least-squares matrix: full

$R\left[F^{2}>2 \sigma\left(F^{2}\right)\right]=0.066$

$w R\left(F^{2}\right)=0.183$

$S=1.05$

4390 reflections

329 parameters

30 restraints
$D_{\mathrm{x}}=1.295 \mathrm{Mg} \mathrm{m}^{-3}$

Mo $K \alpha$ radiation, $\lambda=0.71073 \AA$

Cell parameters from 2016 reflections

$\theta=2-20^{\circ}$

$\mu=0.18 \mathrm{~mm}^{-1}$

$T=298 \mathrm{~K}$

Block, colourless

$0.3 \times 0.3 \times 0.2 \mathrm{~mm}$

$\theta_{\max }=24.9^{\circ}, \theta_{\min }=1.7^{\circ}$

$h=0 \rightarrow 15$

$k=0 \rightarrow 15$

$l=-35 \rightarrow 35$

1 standard reflections every 60 reflections

intensity decay: $1 \%$

Hydrogen site location: mixed

$\mathrm{H}$-atom parameters constrained

$w=1 /\left[\sigma^{2}\left(F_{\mathrm{o}}^{2}\right)+(0.0992 P)^{2}\right]$

where $P=\left(F_{\mathrm{o}}^{2}+2 F_{\mathrm{c}}^{2}\right) / 3$

$(\Delta / \sigma)_{\max }<0.001$

$\Delta \rho_{\max }=0.62 \mathrm{e} \AA^{-3}$

$\Delta \rho_{\min }=-0.30 \mathrm{e} \AA^{-3}$ 
Absolute structure: Flack $x$ determined using 833 quotients $\left[\left(I^{+}\right)-(I)\right] /\left[\left(I^{+}\right)+\left(I^{-}\right)\right]$(Parsons et al., 2013)

Absolute structure parameter: $0.15(8)$

\section{Special details}

Geometry. All esds (except the esd in the dihedral angle between two 1.s. planes) are estimated using the full covariance matrix. The cell esds are taken into account individually in the estimation of esds in distances, angles and torsion angles; correlations between esds in cell parameters are only used when they are defined by crystal symmetry. An approximate (isotropic) treatment of cell esds is used for estimating esds involving l.s. planes.

Fractional atomic coordinates and isotropic or equivalent isotropic displacement parameters $\left(\AA^{2}\right)$

\begin{tabular}{|c|c|c|c|c|c|}
\hline & $x$ & $y$ & $z$ & $U_{\text {iso }} * / U_{\text {eq }}$ & Occ. $(<1)$ \\
\hline S1 & $0.89768(15)$ & $0.65667(14)$ & $0.46183(6)$ & $0.0537(5)$ & \\
\hline $\mathrm{O} 3$ & $0.9782(4)$ & $0.7422(4)$ & $0.33313(15)$ & $0.0565(12)$ & \\
\hline O6 & $1.2492(4)$ & $1.0317(4)$ & $0.51740(15)$ & $0.0650(15)$ & \\
\hline O5 & $0.8948(4)$ & $0.9600(4)$ & $0.44709(16)$ & $0.0618(14)$ & \\
\hline O1 & $0.8059(4)$ & $0.7045(4)$ & $0.44502(17)$ & $0.0690(14)$ & \\
\hline $\mathrm{O} 2$ & $0.9386(4)$ & $0.5729(4)$ & $0.43657(18)$ & $0.0728(16)$ & \\
\hline $\mathrm{O} 4$ & $0.6990(5)$ & $0.8281(4)$ & $0.21258(18)$ & $0.0774(16)$ & \\
\hline $\mathrm{O} 7$ & $1.3846(4)$ & $1.0504(4)$ & $0.45344(16)$ & $0.0660(14)$ & \\
\hline N1 & $0.9578(4)$ & $0.8403(4)$ & $0.39684(16)$ & $0.0425(12)$ & \\
\hline $\mathrm{N} 2$ & $0.8221(4)$ & $0.8054(5)$ & $0.26522(17)$ & $0.0544(15)$ & \\
\hline $\mathrm{H} 2$ & 0.8764 & 0.7699 & 0.2710 & $0.065^{*}$ & \\
\hline $\mathrm{C} 3$ & $0.9329(5)$ & $0.8089(5)$ & $0.3537(2)$ & $0.0418(14)$ & \\
\hline $\mathrm{C} 2$ & $1.0379(5)$ & $0.7928(5)$ & $0.4242(2)$ & $0.0441(15)$ & \\
\hline $\mathrm{H} 2 \mathrm{~A}$ & 1.0637 & 0.7333 & 0.4075 & $0.053 *$ & \\
\hline $\mathrm{C} 12$ & $0.8912(5)$ & $0.9175(5)$ & $0.4107(2)$ & $0.0487(16)$ & \\
\hline $\mathrm{C} 11$ & $0.8173(5)$ & $0.9376(5)$ & $0.3739(2)$ & $0.0452(15)$ & \\
\hline $\mathrm{C} 13$ & $1.1290(5)$ & $0.8638(5)$ & $0.4319(2)$ & $0.0455(16)$ & \\
\hline $\mathrm{C} 14$ & $1.1440(5)$ & $0.9163(5)$ & $0.4720(2)$ & $0.0512(17)$ & \\
\hline H14 & 1.0952 & 0.9100 & 0.4945 & $0.061^{*}$ & \\
\hline $\mathrm{C} 4$ & $0.8437(5)$ & $0.8713(5)$ & $0.3392(2)$ & $0.0451(16)$ & \\
\hline $\mathrm{C} 8$ & $0.7059(5)$ & $0.9390(5)$ & $0.2964(3)$ & $0.0572(18)$ & \\
\hline $\mathrm{H} 8$ & 0.6670 & 0.9408 & 0.2703 & $0.069^{*}$ & \\
\hline $\mathrm{C} 5$ & $0.7893(5)$ & $0.8709(5)$ & $0.2996(2)$ & $0.0477(16)$ & \\
\hline $\mathrm{C} 1$ & $0.9941(5)$ & $0.7515(5)$ & $0.4684(2)$ & $0.0467(15)$ & \\
\hline H1A & 0.9657 & 0.8086 & 0.4853 & $0.056^{*}$ & \\
\hline H1B & 1.0501 & 0.7225 & 0.4858 & $0.056^{*}$ & \\
\hline $\mathrm{C} 20$ & $1.2874(5)$ & $0.9370(6)$ & $0.4048(2)$ & $0.0575(18)$ & \\
\hline $\mathrm{H} 20$ & 1.3353 & 0.9435 & 0.3819 & $0.069^{*}$ & \\
\hline C6 & $0.7810(7)$ & $0.7888(6)$ & $0.2243(2)$ & $0.0599(19)$ & \\
\hline $\mathrm{C} 18$ & $1.3020(5)$ & $0.9885(5)$ & $0.4443(3)$ & $0.0533(18)$ & \\
\hline $\mathrm{C} 15$ & $1.2291(5)$ & $0.9771(5)$ & $0.4791(2)$ & $0.0504(17)$ & \\
\hline $\mathrm{C} 21$ & $1.2011(6)$ & $0.8752(5)$ & $0.3988(2)$ & $0.0571(19)$ & \\
\hline $\mathrm{H} 21$ & 1.1921 & 0.8409 & 0.3718 & $0.069^{*}$ & \\
\hline $\mathrm{C} 10$ & $0.7368(5)$ & $1.0042(6)$ & $0.3713(3)$ & $0.0585(18)$ & \\
\hline H10 & 0.7199 & 1.0481 & 0.3947 & $0.070 *$ & \\
\hline
\end{tabular}




\begin{tabular}{|c|c|c|c|c|c|}
\hline $\mathrm{C} 16$ & $1.1741(5)$ & $1.0248(6)$ & $0.5523(2)$ & $0.065(2)$ & \\
\hline H16A & 1.1668 & 0.9539 & 0.5619 & $0.078^{*}$ & \\
\hline H16B & 1.1078 & 1.0488 & 0.5417 & $0.078^{*}$ & \\
\hline C9 & $0.6809(6)$ & $1.0032(6)$ & $0.3313(3)$ & $0.067(2)$ & \\
\hline H9 & 0.6249 & 1.0473 & 0.3282 & $0.081^{*}$ & \\
\hline $\mathrm{C} 7$ & $0.8411(6)$ & $0.7188(7)$ & 0.1951 (2) & $0.076(2)$ & \\
\hline $\mathrm{H} 7 \mathrm{~A}$ & 0.9067 & 0.7050 & 0.2086 & $0.114^{*}$ & \\
\hline H7B & 0.8513 & 0.7508 & 0.1665 & $0.114 *$ & \\
\hline $\mathrm{H} 7 \mathrm{C}$ & 0.8043 & 0.6553 & 0.1912 & $0.114 *$ & \\
\hline $\mathrm{C} 22$ & $0.8740(7)$ & $0.6177(7)$ & $0.5166(2)$ & 0.083 & \\
\hline $\mathrm{H} 22 \mathrm{~A}$ & 0.8596 & 0.6769 & 0.5347 & $0.124 *$ & \\
\hline $\mathrm{H} 22 \mathrm{~B}$ & 0.9334 & 0.5827 & 0.5281 & $0.124 *$ & \\
\hline $\mathrm{H} 22 \mathrm{C}$ & 0.8159 & 0.5720 & 0.5171 & $0.124 *$ & \\
\hline C19 & $1.4549(6)$ & $1.0668(8)$ & $0.4174(3)$ & $0.086(3)$ & \\
\hline H19A & 1.4204 & 1.1022 & 0.3936 & $0.128^{*}$ & \\
\hline H19B & 1.4798 & 1.0017 & 0.4068 & $0.128^{*}$ & \\
\hline H19C & 1.5118 & 1.1076 & 0.4277 & $0.128 *$ & \\
\hline $\mathrm{C} 17$ & $1.2096(8)$ & $1.0901(9)$ & 0.5904 (3) & $0.104(4)$ & \\
\hline H17A & 1.2163 & 1.1602 & 0.5807 & $0.157^{*}$ & \\
\hline H17B & 1.2750 & 1.0656 & 0.6009 & $0.157^{*}$ & \\
\hline $\mathrm{H} 17 \mathrm{C}$ & 1.1602 & 1.0866 & 0.6142 & $0.157^{*}$ & \\
\hline O8 & $0.4584(16)$ & $0.3998(17)$ & $0.4571(7)$ & $0.177(8)$ & 0.5 \\
\hline H8A & 0.5022 & 0.4544 & 0.4613 & $0.265^{*}$ & 0.5 \\
\hline $\mathrm{C} 23$ & $0.3896(19)$ & $0.3736(17)$ & $0.4889(8)$ & $0.108(7)$ & 0.5 \\
\hline $\mathrm{H} 23 \mathrm{~A}$ & 0.3470 & 0.4341 & 0.4933 & $0.130^{*}$ & 0.5 \\
\hline $\mathrm{H} 23 \mathrm{~B}$ & 0.4291 & 0.3653 & 0.5161 & $0.130^{*}$ & 0.5 \\
\hline $\mathrm{C} 24$ & $0.319(3)$ & $0.287(3)$ & $0.4878(12)$ & $0.164(13)$ & 0.5 \\
\hline $\mathrm{H} 24 \mathrm{~A}$ & 0.3262 & 0.2506 & 0.4601 & $0.247 *$ & 0.5 \\
\hline $\mathrm{H} 24 \mathrm{~B}$ & 0.2492 & 0.3117 & 0.4904 & $0.247 *$ & 0.5 \\
\hline $\mathrm{H} 24 \mathrm{C}$ & 0.3334 & 0.2412 & 0.5122 & $0.247 *$ & 0.5 \\
\hline O9 & $0.6400(13)$ & $0.5606(12)$ & $0.4753(9)$ & $0.192(11)$ & 0.5 \\
\hline H9A & 0.6367 & 0.6301 & 0.4706 & $0.289^{*}$ & 0.5 \\
\hline H9B & 0.7011 & 0.5451 & 0.4635 & $0.289 *$ & 0.5 \\
\hline
\end{tabular}

Atomic displacement parameters $\left(\AA^{2}\right)$

\begin{tabular}{lllllll}
\hline & $U^{11}$ & $U^{22}$ & $U^{33}$ & $U^{12}$ & $U^{13}$ & $U^{23}$ \\
\hline S1 & $0.0592(11)$ & $0.0558(11)$ & $0.0461(9)$ & $-0.0131(9)$ & $-0.0010(8)$ & $0.0016(8)$ \\
O3 & $0.057(3)$ & $0.067(3)$ & $0.045(3)$ & $0.013(2)$ & $0.002(2)$ & $-0.015(2)$ \\
O6 & $0.060(3)$ & $0.082(4)$ & $0.053(3)$ & $-0.023(3)$ & $0.007(2)$ & $-0.021(3)$ \\
O5 & $0.081(3)$ & $0.062(3)$ & $0.043(3)$ & $0.003(3)$ & $0.006(2)$ & $-0.014(2)$ \\
O1 & $0.058(3)$ & $0.083(4)$ & $0.066(3)$ & $-0.008(3)$ & $-0.010(3)$ & $0.005(3)$ \\
O2 & $0.085(4)$ & $0.058(3)$ & $0.076(4)$ & $-0.012(3)$ & $0.002(3)$ & $-0.017(3)$ \\
O4 & $0.079(4)$ & $0.092(4)$ & $0.062(3)$ & $0.015(3)$ & $-0.021(3)$ & $-0.006(3)$ \\
O7 & $0.054(3)$ & $0.080(4)$ & $0.064(3)$ & $-0.019(3)$ & $0.007(3)$ & $-0.004(3)$ \\
N1 & $0.049(3)$ & $0.043(3)$ & $0.036(3)$ & $0.000(3)$ & $0.004(2)$ & $-0.007(2)$ \\
N2 & $0.057(4)$ & $0.064(4)$ & $0.043(3)$ & $0.015(3)$ & $-0.006(3)$ & $-0.008(3)$ \\
C3 & $0.042(3)$ & $0.046(4)$ & $0.037(3)$ & $-0.003(3)$ & $0.009(3)$ & $-0.002(3)$
\end{tabular}




$\begin{array}{lllllll}\text { C2 } & 0.049(4) & 0.048(4) & 0.035(3) & 0.000(3) & 0.000(3) & -0.005(3) \\ \text { C12 } & 0.059(4) & 0.045(4) & 0.041(4) & -0.010(3) & 0.008(3) & -0.003(3) \\ \text { C11 } & 0.048(4) & 0.043(4) & 0.044(4) & -0.002(3) & 0.008(3) & -0.006(3) \\ \text { C13 } & 0.047(4) & 0.045(4) & 0.044(4) & 0.000(3) & 0.003(3) & 0.000(3) \\ \text { C14 } & 0.051(4) & 0.052(4) & 0.051(4) & -0.008(3) & 0.013(3) & -0.004(3) \\ \text { C4 } & 0.051(4) & 0.042(4) & 0.042(4) & -0.006(3) & 0.011(3) & -0.001(3) \\ \text { C8 } & 0.054(4) & 0.054(4) & 0.063(5) & 0.003(4) & -0.005(4) & -0.003(4) \\ \text { C5 } & 0.050(4) & 0.046(4) & 0.048(4) & -0.001(3) & 0.003(3) & 0.001(3) \\ \text { C1 } & 0.049(4) & 0.050(4) & 0.040(3) & -0.007(3) & -0.001(3) & -0.004(3) \\ \text { C20 } & 0.051(4) & 0.069(5) & 0.053(4) & -0.007(4) & 0.013(4) & 0.006(4) \\ \text { C6 } & 0.070(5) & 0.068(5) & 0.042(4) & -0.008(4) & -0.001(4) & 0.002(4) \\ \text { C18 } & 0.046(4) & 0.055(4) & 0.059(4) & -0.005(4) & 0.002(3) & 0.003(3) \\ \text { C15 } & 0.041(4) & 0.055(4) & 0.055(4) & -0.003(3) & -0.001(3) & -0.003(3) \\ \text { C21 } & 0.063(5) & 0.065(5) & 0.043(4) & -0.006(4) & 0.005(3) & -0.006(3) \\ \text { C10 } & 0.059(5) & 0.053(4) & 0.063(5) & 0.010(4) & 0.009(4) & -0.010(4) \\ \text { C16 } & 0.044(4) & 0.090(6) & 0.062(5) & -0.006(4) & 0.004(3) & -0.021(4) \\ \text { C9 } & 0.053(5) & 0.059(5) & 0.090(6) & 0.011(4) & 0.007(4) & -0.001(4) \\ \text { C7 } & 0.079(5) & 0.098(6) & 0.051(4) & 0.024(5) & -0.009(4) & -0.017(4) \\ \text { C22 } & 0.102(7) & 0.096(7) & 0.050(5) & -0.029(5) & 0.010(4) & 0.017(4) \\ \text { C19 } & 0.062(5) & 0.123(8) & 0.072(6) & -0.027(5) & 0.004(4) & 0.023(5) \\ \text { C17 } & 0.086(7) & 0.148(9) & 0.079(7) & -0.024(7) & 0.014(5) & -0.052(6) \\ \text { O8 } & 0.161(12) & 0.190(14) & 0.180(14) & 0.097(10) & -0.023(11) & 0.023(12) \\ \text { C23 } & 0.110(11) & 0.086(10) & 0.129(13) & 0.061(8) & -0.053(9) & 0.002(10) \\ \text { C24 } & 0.179(19) & 0.150(17) & 0.16(2) & 0.014(15) & -0.043(16) & -0.010(15) \\ \text { O9 } & 0.126(14) & 0.125(14) & 0.33(3) & -0.030(11) & 0.032(16) & 0.084(16) \\ & & & & & & \end{array}$

Geometric parameters $\left(\AA,{ }^{\circ}\right)$

\begin{tabular}{llll}
\hline $\mathrm{S} 1-\mathrm{O} 1$ & $1.436(5)$ & $\mathrm{C} 20-\mathrm{H} 20$ & 0.9300 \\
$\mathrm{~S} 1-\mathrm{O} 2$ & $1.428(6)$ & $\mathrm{C} 20-\mathrm{C} 18$ & $1.372(10)$ \\
$\mathrm{S} 1-\mathrm{C} 1$ & $1.768(6)$ & $\mathrm{C} 20-\mathrm{C} 21$ & $1.390(10)$ \\
$\mathrm{S} 1-\mathrm{C} 22$ & $1.744(7)$ & $\mathrm{C} 6-\mathrm{C} 7$ & $1.485(10)$ \\
$\mathrm{O} 3-\mathrm{C} 3$ & $1.214(7)$ & $\mathrm{C} 18-\mathrm{C} 15$ & $1.417(10)$ \\
$\mathrm{O} 6-\mathrm{C} 15$ & $1.373(8)$ & $\mathrm{C} 21-\mathrm{H} 21$ & 0.9300 \\
$\mathrm{O} 6-\mathrm{C} 16$ & $1.433(8)$ & $\mathrm{C} 10-\mathrm{H} 10$ & 0.9300 \\
$\mathrm{O} 5-\mathrm{C} 12$ & $1.221(7)$ & $\mathrm{C} 10-\mathrm{C} 9$ & $1.400(11)$ \\
$\mathrm{O} 4-\mathrm{C} 6$ & $1.232(9)$ & $\mathrm{C} 16-\mathrm{H} 16 \mathrm{~A}$ & 0.9700 \\
$\mathrm{O} 7-\mathrm{C} 18$ & $1.368(8)$ & $\mathrm{C} 16-\mathrm{H} 16 \mathrm{~B}$ & 0.9700 \\
$\mathrm{O} 7-\mathrm{C} 19$ & $1.430(9)$ & $\mathrm{C} 16-\mathrm{C} 17$ & $1.495(11)$ \\
$\mathrm{N} 1-\mathrm{C} 3$ & $1.394(8)$ & $\mathrm{C} 9-\mathrm{H} 9$ & 0.9300 \\
$\mathrm{~N} 1-\mathrm{C} 2$ & $1.461(8)$ & $\mathrm{C} 7-\mathrm{H} 7 \mathrm{~A}$ & 0.9601 \\
$\mathrm{~N} 1-\mathrm{C} 12$ & $1.388(8)$ & $\mathrm{C} 7-\mathrm{H} 7 \mathrm{~B}$ & 0.9599 \\
$\mathrm{~N} 2-\mathrm{H} 2$ & 0.8600 & $\mathrm{C} 7-\mathrm{H} 7 \mathrm{C}$ & 0.9600 \\
$\mathrm{~N} 2-\mathrm{C} 5$ & $1.401(8)$ & $\mathrm{C} 22-\mathrm{H} 22 \mathrm{~A}$ & 0.9600 \\
$\mathrm{~N} 2-\mathrm{C} 6$ & $1.353(9)$ & $\mathrm{C} 22-\mathrm{H} 22 \mathrm{~B}$ & 0.9600 \\
$\mathrm{C} 3-\mathrm{C} 4$ & $1.478(9)$ & $\mathrm{C} 22-\mathrm{H} 22 \mathrm{C}$ & 0.9600 \\
$\mathrm{C} 2-\mathrm{H} 2 \mathrm{~A}$ & 0.9800 & $\mathrm{C} 19-\mathrm{H} 19 \mathrm{~A}$ & 0.9600 \\
$\mathrm{C} 2-\mathrm{C} 13$ & $1.518(9)$ & $\mathrm{C} 19-\mathrm{H} 19 \mathrm{~B}$ &
\end{tabular}




\begin{tabular}{|c|c|c|c|}
\hline $\mathrm{C} 2-\mathrm{C} 1$ & $1.539(8)$ & $\mathrm{C} 19-\mathrm{H} 19 \mathrm{C}$ & 0.9600 \\
\hline $\mathrm{C} 12-\mathrm{C} 11$ & $1.485(9)$ & C17-H17A & 0.9600 \\
\hline $\mathrm{C} 11-\mathrm{C} 4$ & $1.392(8)$ & C17-H17B & 0.9600 \\
\hline $\mathrm{C} 11-\mathrm{C} 10$ & $1.360(9)$ & $\mathrm{C} 17-\mathrm{H} 17 \mathrm{C}$ & 0.9600 \\
\hline $\mathrm{C} 13-\mathrm{C} 14$ & $1.394(9)$ & $\mathrm{O} 8-\mathrm{H} 8 \mathrm{~A}$ & 0.9182 \\
\hline $\mathrm{C} 13-\mathrm{C} 21$ & $1.372(9)$ & $\mathrm{O} 8-\mathrm{C} 23$ & $1.35(2)$ \\
\hline $\mathrm{C} 14-\mathrm{H} 14$ & 0.9300 & $\mathrm{C} 23-\mathrm{H} 23 \mathrm{~A}$ & 0.9700 \\
\hline $\mathrm{C} 14-\mathrm{C} 15$ & $1.375(9)$ & $\mathrm{C} 23-\mathrm{H} 23 \mathrm{~B}$ & 0.9700 \\
\hline $\mathrm{C} 4-\mathrm{C} 5$ & $1.381(9)$ & $\mathrm{C} 23-\mathrm{C} 24$ & $1.46(2)$ \\
\hline $\mathrm{C} 8-\mathrm{H} 8$ & 0.9300 & $\mathrm{C} 24-\mathrm{H} 24 \mathrm{~A}$ & 0.9600 \\
\hline $\mathrm{C} 8-\mathrm{C} 5$ & $1.401(9)$ & $\mathrm{C} 24-\mathrm{H} 24 \mathrm{~B}$ & 0.9600 \\
\hline $\mathrm{C} 8-\mathrm{C} 9$ & $1.377(10)$ & $\mathrm{C} 24-\mathrm{H} 24 \mathrm{C}$ & 0.9600 \\
\hline $\mathrm{C} 1-\mathrm{H} 1 \mathrm{~A}$ & 0.9700 & $\mathrm{O} 9-\mathrm{H} 9 \mathrm{~A}$ & 0.9150 \\
\hline $\mathrm{C} 1-\mathrm{H} 1 \mathrm{~B}$ & 0.9700 & $\mathrm{O} 9-\mathrm{H} 9 \mathrm{~B}$ & 0.8918 \\
\hline $\mathrm{O} 1-\mathrm{S} 1-\mathrm{C} 1$ & $109.0(3)$ & $\mathrm{C} 20-\mathrm{C} 18-\mathrm{C} 15$ & $119.4(6)$ \\
\hline $\mathrm{O} 1-\mathrm{S} 1-\mathrm{C} 22$ & $108.0(4)$ & $\mathrm{O} 6-\mathrm{C} 15-\mathrm{C} 14$ & $125.3(6)$ \\
\hline $\mathrm{O} 2-\mathrm{S} 1-\mathrm{O} 1$ & $117.0(3)$ & $\mathrm{O} 6-\mathrm{C} 15-\mathrm{C} 18$ & $115.7(6)$ \\
\hline $\mathrm{O} 2-\mathrm{S} 1-\mathrm{C} 1$ & $109.1(3)$ & $\mathrm{C} 14-\mathrm{C} 15-\mathrm{C} 18$ & $118.9(6)$ \\
\hline $\mathrm{O} 2-\mathrm{S} 1-\mathrm{C} 22$ & $110.0(4)$ & $\mathrm{C} 13-\mathrm{C} 21-\mathrm{C} 20$ & $121.3(6)$ \\
\hline $\mathrm{C} 22-\mathrm{S} 1-\mathrm{C} 1$ & $102.8(4)$ & $\mathrm{C} 13-\mathrm{C} 21-\mathrm{H} 21$ & 119.3 \\
\hline $\mathrm{C} 15-\mathrm{O} 6-\mathrm{C} 16$ & $116.6(5)$ & $\mathrm{C} 20-\mathrm{C} 21-\mathrm{H} 21$ & 119.3 \\
\hline $\mathrm{C} 18-\mathrm{O} 7-\mathrm{C} 19$ & $115.9(6)$ & $\mathrm{C} 11-\mathrm{C} 10-\mathrm{H} 10$ & 121.9 \\
\hline $\mathrm{C} 3-\mathrm{N} 1-\mathrm{C} 2$ & $124.2(5)$ & $\mathrm{C} 11-\mathrm{C} 10-\mathrm{C} 9$ & $116.3(6)$ \\
\hline $\mathrm{C} 12-\mathrm{N} 1-\mathrm{C} 3$ & $110.2(5)$ & $\mathrm{C} 9-\mathrm{C} 10-\mathrm{H} 10$ & 121.9 \\
\hline $\mathrm{C} 12-\mathrm{N} 1-\mathrm{C} 2$ & $125.5(5)$ & $\mathrm{O} 6-\mathrm{C} 16-\mathrm{H} 16 \mathrm{~A}$ & 110.1 \\
\hline $\mathrm{C} 5-\mathrm{N} 2-\mathrm{H} 2$ & 115.3 & $\mathrm{O} 6-\mathrm{C} 16-\mathrm{H} 16 \mathrm{~B}$ & 110.1 \\
\hline $\mathrm{C} 6-\mathrm{N} 2-\mathrm{H} 2$ & 114.8 & $\mathrm{O} 6-\mathrm{C} 16-\mathrm{C} 17$ & $108.2(6)$ \\
\hline $\mathrm{C} 6-\mathrm{N} 2-\mathrm{C} 5$ & $129.9(6)$ & $\mathrm{H} 16 \mathrm{~A}-\mathrm{C} 16-\mathrm{H} 16 \mathrm{~B}$ & 108.4 \\
\hline $\mathrm{O} 3-\mathrm{C} 3-\mathrm{N} 1$ & $124.4(6)$ & $\mathrm{C} 17-\mathrm{C} 16-\mathrm{H} 16 \mathrm{~A}$ & 110.1 \\
\hline $\mathrm{O} 3-\mathrm{C} 3-\mathrm{C} 4$ & $128.5(6)$ & $\mathrm{C} 17-\mathrm{C} 16-\mathrm{H} 16 \mathrm{~B}$ & 110.1 \\
\hline $\mathrm{N} 1-\mathrm{C} 3-\mathrm{C} 4$ & $107.0(5)$ & $\mathrm{C} 8-\mathrm{C} 9-\mathrm{C} 10$ & $122.2(7)$ \\
\hline $\mathrm{N} 1-\mathrm{C} 2-\mathrm{H} 2 \mathrm{~A}$ & 106.9 & $\mathrm{C} 8-\mathrm{C} 9-\mathrm{H} 9$ & 118.9 \\
\hline $\mathrm{N} 1-\mathrm{C} 2-\mathrm{C} 13$ & $112.7(5)$ & $\mathrm{C} 10-\mathrm{C} 9-\mathrm{H} 9$ & 118.9 \\
\hline $\mathrm{N} 1-\mathrm{C} 2-\mathrm{C} 1$ & $111.5(5)$ & $\mathrm{C} 6-\mathrm{C} 7-\mathrm{H} 7 \mathrm{~A}$ & 109.4 \\
\hline $\mathrm{C} 13-\mathrm{C} 2-\mathrm{H} 2 \mathrm{~A}$ & 106.9 & $\mathrm{C} 6-\mathrm{C} 7-\mathrm{H} 7 \mathrm{~B}$ & 109.4 \\
\hline $\mathrm{C} 13-\mathrm{C} 2-\mathrm{C} 1$ & $111.6(5)$ & $\mathrm{C} 6-\mathrm{C} 7-\mathrm{H} 7 \mathrm{C}$ & 109.6 \\
\hline $\mathrm{C} 1-\mathrm{C} 2-\mathrm{H} 2 \mathrm{~A}$ & 106.9 & $\mathrm{H} 7 \mathrm{~A}-\mathrm{C} 7-\mathrm{H} 7 \mathrm{~B}$ & 109.5 \\
\hline $\mathrm{O} 5-\mathrm{C} 12-\mathrm{N} 1$ & $124.8(6)$ & $\mathrm{H} 7 \mathrm{~A}-\mathrm{C} 7-\mathrm{H} 7 \mathrm{C}$ & 109.5 \\
\hline $\mathrm{O} 5-\mathrm{C} 12-\mathrm{C} 11$ & $127.4(6)$ & $\mathrm{H} 7 \mathrm{~B}-\mathrm{C} 7-\mathrm{H} 7 \mathrm{C}$ & 109.4 \\
\hline $\mathrm{N} 1-\mathrm{C} 12-\mathrm{C} 11$ & $107.9(5)$ & $\mathrm{S} 1-\mathrm{C} 22-\mathrm{H} 22 \mathrm{~A}$ & 109.5 \\
\hline $\mathrm{C} 4-\mathrm{C} 11-\mathrm{C} 12$ & $106.6(6)$ & $\mathrm{S} 1-\mathrm{C} 22-\mathrm{H} 22 \mathrm{~B}$ & 109.5 \\
\hline $\mathrm{C} 10-\mathrm{C} 11-\mathrm{C} 12$ & $130.7(6)$ & $\mathrm{S} 1-\mathrm{C} 22-\mathrm{H} 22 \mathrm{C}$ & 109.5 \\
\hline $\mathrm{C} 10-\mathrm{C} 11-\mathrm{C} 4$ & $122.7(6)$ & $\mathrm{H} 22 \mathrm{~A}-\mathrm{C} 22-\mathrm{H} 22 \mathrm{~B}$ & 109.5 \\
\hline $\mathrm{C} 14-\mathrm{C} 13-\mathrm{C} 2$ & $122.5(5)$ & $\mathrm{H} 22 \mathrm{~A}-\mathrm{C} 22-\mathrm{H} 22 \mathrm{C}$ & 109.5 \\
\hline $\mathrm{C} 21-\mathrm{C} 13-\mathrm{C} 2$ & $119.2(6)$ & $\mathrm{H} 22 \mathrm{~B}-\mathrm{C} 22-\mathrm{H} 22 \mathrm{C}$ & 109.5 \\
\hline $\mathrm{C} 21-\mathrm{C} 13-\mathrm{C} 14$ & $118.3(6)$ & $\mathrm{O} 7-\mathrm{C} 19-\mathrm{H} 19 \mathrm{~A}$ & 109.5 \\
\hline $\mathrm{C} 13-\mathrm{C} 14-\mathrm{H} 14$ & 119.1 & O7-C19-H19B & 109.5 \\
\hline
\end{tabular}




\begin{tabular}{|c|c|c|c|}
\hline $\mathrm{C} 15-\mathrm{C} 14-\mathrm{C} 13$ & $121.8(6)$ & $\mathrm{O} 7-\mathrm{C} 19-\mathrm{H} 19 \mathrm{C}$ & 109.5 \\
\hline $\mathrm{C} 15-\mathrm{C} 14-\mathrm{H} 14$ & 119.1 & $\mathrm{H} 19 \mathrm{~A}-\mathrm{C} 19-\mathrm{H} 19 \mathrm{~B}$ & 109.5 \\
\hline $\mathrm{C} 11-\mathrm{C} 4-\mathrm{C} 3$ & $108.3(6)$ & $\mathrm{H} 19 \mathrm{~A}-\mathrm{C} 19-\mathrm{H} 19 \mathrm{C}$ & 109.5 \\
\hline $\mathrm{C} 5-\mathrm{C} 4-\mathrm{C} 3$ & $130.6(6)$ & $\mathrm{H} 19 \mathrm{~B}-\mathrm{C} 19-\mathrm{H} 19 \mathrm{C}$ & 109.5 \\
\hline $\mathrm{C} 5-\mathrm{C} 4-\mathrm{C} 11$ & $121.1(6)$ & $\mathrm{C} 16-\mathrm{C} 17-\mathrm{H} 17 \mathrm{~A}$ & 109.5 \\
\hline $\mathrm{C} 5-\mathrm{C} 8-\mathrm{H} 8$ & 119.6 & $\mathrm{C} 16-\mathrm{C} 17-\mathrm{H} 17 \mathrm{~B}$ & 109.5 \\
\hline $\mathrm{C} 9-\mathrm{C} 8-\mathrm{H} 8$ & 119.6 & $\mathrm{C} 16-\mathrm{C} 17-\mathrm{H} 17 \mathrm{C}$ & 109.5 \\
\hline $\mathrm{C} 9-\mathrm{C} 8-\mathrm{C} 5$ & $120.8(7)$ & $\mathrm{H} 17 \mathrm{~A}-\mathrm{C} 17-\mathrm{H} 17 \mathrm{~B}$ & 109.5 \\
\hline $\mathrm{N} 2-\mathrm{C} 5-\mathrm{C} 8$ & $124.6(6)$ & $\mathrm{H} 17 \mathrm{~A}-\mathrm{C} 17-\mathrm{H} 17 \mathrm{C}$ & 109.5 \\
\hline $\mathrm{C} 4-\mathrm{C} 5-\mathrm{N} 2$ & $118.5(6)$ & $\mathrm{H} 17 \mathrm{~B}-\mathrm{C} 17-\mathrm{H} 17 \mathrm{C}$ & 109.5 \\
\hline $\mathrm{C} 4-\mathrm{C} 5-\mathrm{C} 8$ & $116.9(6)$ & $\mathrm{C} 23-\mathrm{O} 8-\mathrm{H} 8 \mathrm{~A}$ & 120.7 \\
\hline $\mathrm{S} 1-\mathrm{C} 1-\mathrm{H} 1 \mathrm{~A}$ & 108.7 & $\mathrm{O} 8-\mathrm{C} 23-\mathrm{H} 23 \mathrm{~A}$ & 105.6 \\
\hline $\mathrm{S} 1-\mathrm{C} 1-\mathrm{H} 1 \mathrm{~B}$ & 108.7 & $\mathrm{O} 8-\mathrm{C} 23-\mathrm{H} 23 \mathrm{~B}$ & 105.6 \\
\hline $\mathrm{C} 2-\mathrm{C} 1-\mathrm{S} 1$ & $114.1(4)$ & $\mathrm{O} 8-\mathrm{C} 23-\mathrm{C} 24$ & $127(3)$ \\
\hline $\mathrm{C} 2-\mathrm{C} 1-\mathrm{H} 1 \mathrm{~A}$ & 108.7 & $\mathrm{H} 23 \mathrm{~A}-\mathrm{C} 23-\mathrm{H} 23 \mathrm{~B}$ & 106.1 \\
\hline $\mathrm{C} 2-\mathrm{C} 1-\mathrm{H} 1 \mathrm{~B}$ & 108.7 & $\mathrm{C} 24-\mathrm{C} 23-\mathrm{H} 23 \mathrm{~A}$ & 105.6 \\
\hline $\mathrm{H} 1 \mathrm{~A}-\mathrm{C} 1-\mathrm{H} 1 \mathrm{~B}$ & 107.6 & $\mathrm{C} 24-\mathrm{C} 23-\mathrm{H} 23 \mathrm{~B}$ & 105.6 \\
\hline $\mathrm{C} 18-\mathrm{C} 20-\mathrm{H} 20$ & 119.8 & $\mathrm{C} 23-\mathrm{C} 24-\mathrm{H} 24 \mathrm{~A}$ & 109.5 \\
\hline $\mathrm{C} 18-\mathrm{C} 20-\mathrm{C} 21$ & $120.3(6)$ & $\mathrm{C} 23-\mathrm{C} 24-\mathrm{H} 24 \mathrm{~B}$ & 109.5 \\
\hline $\mathrm{C} 21-\mathrm{C} 20-\mathrm{H} 20$ & 119.8 & $\mathrm{C} 23-\mathrm{C} 24-\mathrm{H} 24 \mathrm{C}$ & 109.5 \\
\hline $\mathrm{O} 4-\mathrm{C} 6-\mathrm{N} 2$ & $122.3(7)$ & $\mathrm{H} 24 \mathrm{~A}-\mathrm{C} 24-\mathrm{H} 24 \mathrm{~B}$ & 109.5 \\
\hline $\mathrm{O} 4-\mathrm{C} 6-\mathrm{C} 7$ & $122.7(7)$ & $\mathrm{H} 24 \mathrm{~A}-\mathrm{C} 24-\mathrm{H} 24 \mathrm{C}$ & 109.5 \\
\hline $\mathrm{N} 2-\mathrm{C} 6-\mathrm{C} 7$ & $115.1(7)$ & $\mathrm{H} 24 \mathrm{~B}-\mathrm{C} 24-\mathrm{H} 24 \mathrm{C}$ & 109.5 \\
\hline $\mathrm{O} 7-\mathrm{C} 18-\mathrm{C} 20$ & $124.7(6)$ & $\mathrm{H} 9 \mathrm{~A}-\mathrm{O} 9-\mathrm{H} 9 \mathrm{~B}$ & 101.7 \\
\hline $\mathrm{O} 7-\mathrm{C} 18-\mathrm{C} 15$ & $116.0(6)$ & & \\
\hline $\mathrm{O} 3-\mathrm{C} 3-\mathrm{C} 4-\mathrm{C} 11$ & $179.6(6)$ & $\mathrm{C} 12-\mathrm{C} 11-\mathrm{C} 10-\mathrm{C} 9$ & $-179.1(7)$ \\
\hline $\mathrm{O} 3-\mathrm{C} 3-\mathrm{C} 4-\mathrm{C} 5$ & $-0.2(11)$ & $\mathrm{C} 11-\mathrm{C} 4-\mathrm{C} 5-\mathrm{N} 2$ & $177.4(6)$ \\
\hline $\mathrm{O} 5-\mathrm{C} 12-\mathrm{C} 11-\mathrm{C} 4$ & $179.6(6)$ & $\mathrm{C} 11-\mathrm{C} 4-\mathrm{C} 5-\mathrm{C} 8$ & $-1.0(10)$ \\
\hline $\mathrm{O} 5-\mathrm{C} 12-\mathrm{C} 11-\mathrm{C} 10$ & $-1.3(12)$ & $\mathrm{C} 11-\mathrm{C} 10-\mathrm{C} 9-\mathrm{C} 8$ & $-0.4(11)$ \\
\hline $\mathrm{O} 1-\mathrm{S} 1-\mathrm{C} 1-\mathrm{C} 2$ & $-71.1(6)$ & $\mathrm{C} 13-\mathrm{C} 2-\mathrm{C} 1-\mathrm{S} 1$ & $-173.0(4)$ \\
\hline $\mathrm{O} 2-\mathrm{S} 1-\mathrm{C} 1-\mathrm{C} 2$ & $57.7(6)$ & $\mathrm{C} 13-\mathrm{C} 14-\mathrm{C} 15-\mathrm{O} 6$ & $179.5(7)$ \\
\hline $\mathrm{O} 7-\mathrm{C} 18-\mathrm{C} 15-\mathrm{O} 6$ & $1.6(9)$ & $\mathrm{C} 13-\mathrm{C} 14-\mathrm{C} 15-\mathrm{C} 18$ & $1.6(10)$ \\
\hline $\mathrm{O} 7-\mathrm{C} 18-\mathrm{C} 15-\mathrm{C} 14$ & $179.7(6)$ & $\mathrm{C} 14-\mathrm{C} 13-\mathrm{C} 21-\mathrm{C} 20$ & $0.2(10)$ \\
\hline $\mathrm{N} 1-\mathrm{C} 3-\mathrm{C} 4-\mathrm{C} 11$ & $-0.2(7)$ & $\mathrm{C} 4-\mathrm{C} 11-\mathrm{C} 10-\mathrm{C} 9$ & $-0.2(10)$ \\
\hline $\mathrm{N} 1-\mathrm{C} 3-\mathrm{C} 4-\mathrm{C} 5$ & $180.0(6)$ & $\mathrm{C} 5-\mathrm{N} 2-\mathrm{C} 6-\mathrm{O} 4$ & $-5.0(12)$ \\
\hline $\mathrm{N} 1-\mathrm{C} 2-\mathrm{C} 13-\mathrm{C} 14$ & $102.5(7)$ & $\mathrm{C} 5-\mathrm{N} 2-\mathrm{C} 6-\mathrm{C} 7$ & $175.8(7)$ \\
\hline $\mathrm{N} 1-\mathrm{C} 2-\mathrm{C} 13-\mathrm{C} 21$ & $-79.2(7)$ & $\mathrm{C} 5-\mathrm{C} 8-\mathrm{C} 9-\mathrm{C} 10$ & $0.3(12)$ \\
\hline $\mathrm{N} 1-\mathrm{C} 2-\mathrm{C} 1-\mathrm{S} 1$ & $60.1(6)$ & $\mathrm{C} 1-\mathrm{C} 2-\mathrm{C} 13-\mathrm{C} 14$ & $-23.8(9)$ \\
\hline $\mathrm{N} 1-\mathrm{C} 12-\mathrm{C} 11-\mathrm{C} 4$ & $-0.3(7)$ & $\mathrm{C} 1-\mathrm{C} 2-\mathrm{C} 13-\mathrm{C} 21$ & $154.5(6)$ \\
\hline $\mathrm{N} 1-\mathrm{C} 12-\mathrm{C} 11-\mathrm{C} 10$ & $178.8(7)$ & $\mathrm{C} 20-\mathrm{C} 18-\mathrm{C} 15-\mathrm{O} 6$ & $-179.6(6)$ \\
\hline $\mathrm{C} 3-\mathrm{N} 1-\mathrm{C} 2-\mathrm{C} 13$ & $111.8(6)$ & $\mathrm{C} 20-\mathrm{C} 18-\mathrm{C} 15-\mathrm{C} 14$ & $-1.5(10)$ \\
\hline $\mathrm{C} 3-\mathrm{N} 1-\mathrm{C} 2-\mathrm{C} 1$ & $-121.8(6)$ & $\mathrm{C} 6-\mathrm{N} 2-\mathrm{C} 5-\mathrm{C} 4$ & $177.9(7)$ \\
\hline $\mathrm{C} 3-\mathrm{N} 1-\mathrm{C} 12-\mathrm{O} 5$ & $-179.8(6)$ & $\mathrm{C} 6-\mathrm{N} 2-\mathrm{C} 5-\mathrm{C} 8$ & $-3.9(12)$ \\
\hline $\mathrm{C} 3-\mathrm{N} 1-\mathrm{C} 12-\mathrm{C} 11$ & $0.1(7)$ & $\mathrm{C} 18-\mathrm{C} 20-\mathrm{C} 21-\mathrm{C} 13$ & $-0.1(11)$ \\
\hline $\mathrm{C} 3-\mathrm{C} 4-\mathrm{C} 5-\mathrm{N} 2$ & $-2.9(11)$ & $\mathrm{C} 15-\mathrm{O} 6-\mathrm{C} 16-\mathrm{C} 17$ & $-179.4(7)$ \\
\hline $\mathrm{C} 3-\mathrm{C} 4-\mathrm{C} 5-\mathrm{C} 8$ & $178.7(6)$ & $\mathrm{C} 21-\mathrm{C} 13-\mathrm{C} 14-\mathrm{C} 15$ & $-1.0(10)$ \\
\hline $\mathrm{C} 2-\mathrm{N} 1-\mathrm{C} 3-\mathrm{O} 3$ & $-3.8(9)$ & $\mathrm{C} 21-\mathrm{C} 20-\mathrm{C} 18-\mathrm{O} 7$ & $179.5(7)$ \\
\hline
\end{tabular}




$\begin{array}{llll}\mathrm{C} 2-\mathrm{N} 1-\mathrm{C} 3-\mathrm{C} 4 & 176.1(5) & \mathrm{C} 21-\mathrm{C} 20-\mathrm{C} 18-\mathrm{C} 15 & 0.8(11) \\ \mathrm{C} 2-\mathrm{N} 1-\mathrm{C} 12-\mathrm{O} 5 & 4.3(10) & \mathrm{C} 10-\mathrm{C} 11-\mathrm{C} 4-\mathrm{C} 3 & -178.9(6) \\ \mathrm{C} 2-\mathrm{N} 1-\mathrm{C} 12-\mathrm{C} 11 & -175.8(5) & \mathrm{C} 10-\mathrm{C} 11-\mathrm{C} 4-\mathrm{C} 5 & 0.9(10) \\ \mathrm{C} 2-\mathrm{C} 13-\mathrm{C} 14-\mathrm{C} 15 & 177.3(6) & \mathrm{C} 16-\mathrm{O} 6-\mathrm{C} 15-\mathrm{C} 14 & -0.5(10) \\ \mathrm{C} 2-\mathrm{C} 13-\mathrm{C} 21-\mathrm{C} 20 & -178.2(6) & \mathrm{C} 16-\mathrm{O} 6-\mathrm{C} 15-\mathrm{C} 18 & 177.4(6) \\ \mathrm{C} 12-\mathrm{N} 1-\mathrm{C} 3-\mathrm{O} 3 & -179.8(6) & \mathrm{C} 9-\mathrm{C} 8-\mathrm{C} 5-\mathrm{N} 2 & -177.9(7) \\ \mathrm{C} 12-\mathrm{N} 1-\mathrm{C} 3-\mathrm{C} 4 & 0.1(6) & \mathrm{C} 9-\mathrm{C} 8-\mathrm{C} 5-\mathrm{C} 4 & 0.4(10) \\ \mathrm{C} 12-\mathrm{N} 1-\mathrm{C} 2-\mathrm{C} 13 & -72.8(7) & \mathrm{C} 22-\mathrm{S} 1-\mathrm{C} 1-\mathrm{C} 2 & 174.5(5) \\ \mathrm{C} 12-\mathrm{N} 1-\mathrm{C} 2-\mathrm{C} 1 & 53.6(8) & \mathrm{C} 19-\mathrm{O} 7-\mathrm{C} 18-\mathrm{C} 20 & 4.8(10) \\ \mathrm{C} 12-\mathrm{C} 11-\mathrm{C} 4-\mathrm{C} 3 & 0.3(7) & \mathrm{C} 19-\mathrm{O} 7-\mathrm{C} 18-\mathrm{C} 15 & -176.4(7) \\ \mathrm{C} 12-\mathrm{C} 11-\mathrm{C} 4-\mathrm{C} 5 & -179.9(6) & & \end{array}$

Hydrogen-bond geometry $\left(\hat{A},{ }^{\circ}\right)$

\begin{tabular}{lllll}
\hline$D-\mathrm{H} \cdots A$ & $D-\mathrm{H}$ & $\mathrm{H} \cdots A$ & $D \cdots A$ & $D-\mathrm{H} \cdots A$ \\
\hline $\mathrm{N} 2-\mathrm{H} 2 \cdots \mathrm{O} 3$ & 0.86 & 2.31 & $2.986(7)$ & 136 \\
$\mathrm{O} 8-\mathrm{H} 8 A \cdots \mathrm{O} 9$ & 0.92 & 2.30 & $3.20(3)$ & 166 \\
$\mathrm{O} 9-\mathrm{H} 9 B \cdots \mathrm{O} 1$ & 0.89 & 2.54 & $2.994(17)$ & 112 \\
$\mathrm{C} 8-\mathrm{H} 8 \cdots \mathrm{O} 4$ & 0.93 & 2.30 & $2.894(9)$ & 121 \\
$\mathrm{C} 1-\mathrm{H} 1 A \cdots \mathrm{O} 5$ & 0.97 & 2.45 & $3.068(8)$ & 121 \\
$\mathrm{C} 1-\mathrm{H} 1 A \cdots \mathrm{O} 5^{\mathrm{i}}$ & 0.97 & 2.32 & $3.172(8)$ & 147 \\
$\mathrm{C} 1-\mathrm{H} 1 B \cdots \mathrm{O} 4^{\mathrm{ii}}$ & 0.97 & 2.56 & $3.524(9)$ & 172 \\
$\mathrm{C} 14-\mathrm{H} 14 \cdots \mathrm{O} 5^{\mathrm{i}}$ & 0.93 & 2.49 & $3.415(8)$ & 178 \\
$\mathrm{C} 19-\mathrm{H} 19 C \cdots \mathrm{O} 4^{\mathrm{iii}}$ & 0.96 & 2.61 & $3.567(10)$ & 173 \\
$\mathrm{C} 20-\mathrm{H} 20 \cdots \mathrm{O} 2^{\mathrm{ii}}$ & 0.93 & 2.46 & $3.370(9)$ & 166 \\
$\mathrm{C} 22-\mathrm{H} 22 C \cdots \mathrm{O} 3^{\text {iv }}$ & 0.96 & 2.44 & $3.088(9)$ & 124 \\
$\mathrm{C} 22-\mathrm{H} 22 C \cdots \mathrm{O} 9$ & 0.96 & 2.61 & $3.36(2)$ & 136 \\
\hline
\end{tabular}

Symmetry codes: (i) $y, x,-z+1$; (ii) $x+1 / 2,-y+3 / 2,-z+3 / 4$; (iii) $-y+5 / 2, x+1 / 2, z+1 / 4$; (iv) $-y+3 / 2, x-1 / 2, z+1 / 4$. 